\title{
Fuentes y encuadres del discurso mediático del acoso escolar en los periódicos El Mundo y El País
}

\section{Sources and frames in the media discourse of school bullying in El Mundo and El País}

\author{
Fernando Sahuquillo Verdet. Universidad CEU Cardenal Herrera (nandosahuquillo@gmail.com)
}

Recibido: 14/10/2016 - Aceptado: 04/10/17

\section{Resumen:}

Partiendo de la capacidad de los medios de comunicación para configurar la agenda pública y la percepción de los temas de interés, esta investigación propone un análisis del tratamiento del fenómeno del acoso escolar de dos diarios de alcance nacional. Siguiendo los planteamientos de la teoría del framing se analizan los textos periodísticos -de septiembre de 2014 a junio de 2015- para identificar cuáles son las principales fuentes y desvelar si los mensajes transmiten una especial representación del fenómeno, indagan en las causas, proponen una evaluación moral o apuntan cuál debería ser su tratamiento en el futuro. Los diarios seleccionados para el estudio han sido El País y El Mundo por ser dos de las principales referencias impresas de alcance nacional. La investigación demuestra que a pesar de tratarse de un fenómeno permanente y continuado el acoso escolar recibe la atención de los medios de comunicación cuando tiene lugar un suceso dramático como la muerte de un adolescente.

Palabras clave:

Acoso escolar; medios de comunicación; encuadres; agenda pública; fuentes.

\begin{abstract}
:
This research outlines an analysis of the treatment of school bullying in two Spanish newspapers based on the ability of the media to set the public agenda and influence the perception of topics of interest. Journalistic texts are analyzed from September 2014 to June 2015 following the approach of framing theory to identify the main sources and reveal whether the messages convey a particular representation of the phenomenon, investigate the causes, suggest a moral evaluation or what its treatment should be in the future. The newspapers we selected for the analysis were El Pais and El Mundo as they are the main national print references. This research shows that despite being an ongoing phenomenon, school bullying only receives media attention when a dramatic event takes place, such as the death of a teenager.
\end{abstract}

Keywords:

Bullying; mass media; framing; public agenda; sources. 


\section{Introducción}

La Fundación Ayuda a Niños y Adolescentes en Riesgo (ANAR) alertó a principios de 2016 del aumento de llamadas telefónicas a su número de atención desde enero de ese año, momento en el que se conoció el suicidio de Diego, un niño de Leganés (Madrid) tras, presuntamente, haber sufrido acoso escolar. Así, mientras que cincuenta días antes de que se conociera el caso se habían producido 72 llamadas, en los 50 días posteriores se registraron 267. Así se recoge en el informe Acoso escolar: I Informe sobre el 'bullying' según los afectados y líneas de actuación, publicado junto a la Fundación Mutua Madrileña. Crecieron las llamadas de padres pidiendo ayuda, de profesores preguntando por protocolos de actuación, de niños y adolescentes que aseguraban sufrir acoso, así como de padres de niños testigos de situaciones de violencia. Por otra parte, dado el notable incremento de contactos desde que se conociera a través de los medios el caso del niño de Leganés, el informe sostiene que las informaciones periodísticas no generan más situaciones de acoso, sino una mayor reacción social ante el problema (Fundación ANAR y Fundación Mutua Madrileña, 2016:9). Este aumento de llamadas invita a pensar que la cobertura genera una mayor reacción social.

Partiendo de la capacidad de los medios de comunicación para configurar la agenda pública, es decir, de proporcionar a la sociedad los temas de interés e, incluso, las claves de pensamiento sobre esos asuntos ya sea mediante la selección de fuentes o mediante determinados enfoques, esta investigación quiere abordar el fenómeno del acoso escolar, precisamente, desde su presencia y tratamiento en la prensa diaria.

Los medios ejercen tres formas de poder: la primera es el poder de establecer, como se ha dicho, la agenda. La segunda es el poder de definir -no el qué sino el cómo- la manera en que se habla de un tema o de un grupo social determinado. La tercera forma en que se puede manifestar el poder de los medios es mediante la representación de la normalidad. La repetición constante de una manera de hablar acerca de un grupo social en los medios puede llevar a considerarla como natural y normal para todos (Morduchowicz, 2003: 7).

Siguiendo estos planteamientos teóricos este trabajo persigue los siguientes objetivos:

O1. Identificar la atención mediática recibida por el fenómeno de la violencia escolar en los periódicos El País y El Mundo desde septiembre de 2014 a junio de 2015.

O2. Revelar las fuentes y los actores dominantes en los textos periodísticos sobre el acoso escolar en el periodo y los medios analizados.

O3. Analizar qué representación del fenómeno del acoso escolar trasladan los textos de información u opinión que lo tratan. 
Estos tres objetivos se relacionan directamente con otras tantas preguntas de investigación:

PI. 1. ¿Va ligada la cobertura del fenómeno del acoso escolar a determinados sucesos como agresiones o suicidios?

PI. 2. ¿Cuáles son las fuentes dominantes en el discurso mediático de El Mundo y El País sobre el acoso escolar en el periodo analizado?

PI. 3. ¿ Promueve el discurso mediático del acoso escolar una determinada representación del fenómeno?

\subsection{Conceptos de acoso y violencia escolar}

A la hora de proponer una definición de acoso escolar la mayoría de los autores y estudios consultados coinciden en tomar como punto de partida la formulada en 1973 por Dan Olweus: "Un estudiante es acosado o victimizado cuando está expuesto de manera repetitiva a acciones negativas por parte de uno o más estudiantes, sin capacidad para defenderse” (citado en Rubio, 2013: 2). A partir de esta propuesta la conceptualización del acoso escolar puede centrarse en la "intencionalidad de los sujetos", de forma que las acciones sean deliberadamente hostiles (Armero, Bernardino y Bonet de Luna, 2011: 662). Centrando la atención en el entorno escolar conviene distinguir, igualmente, el acoso de una pelea entre compañeros. En el caso del acoso se da relación de “sumisión-dominación” (Cepeda y Caicedo, 2013: 2).

Oñate y Piñuel (2007: 52-55) desarrollan en el conocido como Informe Cisneros X el concepto de acoso y violencia escolar (AVE) como la suma de distintos términos y realidades. Así, la violencia y la intimidación físicas sumadas a la violencia verbal y social darían como resultado el AVE. Las prácticas de violencia física equivaldrían a lo que suele conocerse con la voz inglesa bullying, mientras que el resto vendría a recogerse bajo el amplísimo término de violencia psicológica. Igualmente, comparando el fenómeno con un iceberg, la violencia física sería ese $10 \%$ que se ve, mientras que el resto sería esa gran mole de hielo que no se distingue a simple vista pero que provoca daños como el estrés postraumático, somatizaciones, depresión, ansiedad, tendencia al suicidio, cambios de personalidad, baja autoestima, etc.

En términos jurídicos, la Fiscalía General del Estado (2005: 5) destaca el componente de “desequilibrio de poder" por la presión del grupo, la diferencia de fuerza o edad o la discapacidad de la víctima. Concurre, además, una continuidad en el tiempo, pudiendo consistir los actos concretos que lo integran en agresiones físicas, amenazas, vejaciones, coacciones, insultos o en el aislamiento deliberado de la víctima.

\subsubsection{Características de las personas que intervienen}

En el acoso escolar participan diferentes personas que adoptan roles diferenciados y, al mismo tiempo, variables en función del contexto y del momento en el que se produzca (Comunidad de Madrid, 2012: 10-14). Para diferenciar las figuras que se dan y los roles posibles en el denominado "triángulo del acoso" (víctimas, acosadores y espectadores pasivos) es necesario atender a sus perfiles. 


\subsubsection{Víctimas}

Las víctimas de acoso escolar suelen responder a los rasgos que a continuación se relacionan aunque ello no quiere decir que todos los alumnos que presentan estos rasgos sean víctimas de acoso, ni que no pueda serlo cualquier alumno que no los presente:

1. Vulnerabilidad física o psicológica.

2. Estrategias de defensa y modo de afrontar el problema inadecuadas: aislamiento, resignación, asunción de culpabilidad...

3. Baja autoestima.

4. Escasas habilidades sociales, en especial cuando van acompañadas de éxito académico y buenas relaciones con los profesores.

5. Discapacidad.

6. Características personales distintas a las dominantes: obesidad, miopía, forma de vestir tradicional o descuidada, falta de higiene, pertenencia a minorías étnicas o culturales...

\subsubsection{Acosadores}

El acosador suele responder al siguiente perfil aunque, una vez más, ello no significa que todos los alumnos que encajan en él sean necesariamente acosadores, ni que no pueda serlo un alumno que no lo haga.

1. Suele ser físicamente fuerte.

2. Necesita dominar, tener poder, sentirse superior.

3. Posee un fuerte temperamento, fácilmente enojable.

4. Es impulsivo.

5. Manifiesta baja tolerancia a la frustración.

6. Se muestra desafiante y agresivo hacia los adultos.

7. No suele mostrarse ansioso ni inseguro.

8. Exhibe comportamientos antisociales tempranos.

9. Es poco popular entre sus compañeros y compañeras; solo algunos le siguen.

10. Manifiesta actitud negativa hacia la escuela.

11. Sufre de egocentrismo, escasa capacidad de autocrítica y ausencia de sentimiento de culpabilidad por el acoso, del que suele responsabilizar a la víctima.

12. Muestra una clara falta de empatía y de capacidad para percibir el dolor ajeno. 


\subsubsection{Espectadores pasivos}

La influencia que los agresores ejercen sobre los demás se manifiesta en la falta de apoyo de los compañeros hacia la víctima. Regularmente, los actos de esta índole suelen producirse bajo el conocimiento de compañeros que, inducidos por el contagio social que inhibe la ayuda o el miedo a ser incluidos en el círculo de la víctima, no hacen nada aunque sientan que deben hacerlo. Las principales características de estos observadores pasivos son:

1. Son conscientes del maltrato.

2. Lo toleran y se inhiben sistemáticamente cuando se produce.

3. Conocen a todos los sujetos que intervienen (maltratadores y víctimas), así como las circunstancias concretas que lo rodean.

4. Colaboran en el acoso.

5. Graban agresiones.

6. Las aprueban con su presencia.

7. Refuerzan la conducta de quien acosa.

8. Ignoran y aíslan.

\subsection{Alcance del acoso escolar en España}

Aún hoy, casi una década después de que se publicaran, existen dos documentos que siguen siendo un referente cuando se trata de medir el alcance del fenómeno del acoso escolar en España. Son el informe Violencia escolar: el maltrato entre iguales en la Educación Secundaria Obligatoria 1999-2006, elaborado por la oficina del Defensor del Pueblo (2007), y Acoso y violencia escolar en España, publicado en 2007 por Araceli Oñate e Iñaki Piñuel, también conocido como Informe Cisneros X. Ambos estudios, junto al más reciente I Informe sobre el 'bullying’ según los afectados y líneas de actuación (Fundación ANAR y Fundación Mutua Madrileña, 2016), suponen puntos de referencia inevitables para el abordaje de la cuestión del acoso escolar, a pesar de la divergencia, en algunos casos, de las cifras aportadas.

\subsubsection{Violencia escolar: el maltrato entre iguales en la Educación Secundaria Obligatoria 1999-2006}

La muestra del estudio estuvo formada por 3.000 estudiantes de Educación Secundaria Obligatoria pertenecientes a 300 centros educativos públicos, concertados y privados de todo el territorio nacional. Además, se encuestó a 300 profesoras y profesores que ocupaban la jefatura de estudios en cada uno de dichos centros.

Sobre los resultados del informe, vale la pena detenerse en la frecuencia con la que aparecen en los centros educativos las distintas conductas que suponen maltrato entre iguales por abuso de poder, como su evolución en los cursos de la educación secundaria. En este sentido, siguiendo los resultados del informe, algo más de un 2\% de los alumnos de Secundaria 
asegura sufrir a veces agresiones verbales de algún tipo (motes o hablar mal). Sin embargo, en el caso de los insultos, el $3,9 \%$ dice que ocurre en muchos casos, así como el 5,2\% en los motes o el 4,2\% que señala que hablan mal. Es decir, las prácticas habituales son las agresiones verbales de algún tipo.

Por otra parte, se observa una constante general en cuanto a las prácticas ofensivas a lo largo de los cuatro cursos de Educación Secundaria Obligatoria, con algunas excepciones. Se detecta un descenso notable del primero al cuarto curso de la utilización de motes e insultos. Igualmente, desciende el porcentaje de alumnos que asegura que sus compañeros "no les dejan participar". También es considerable el descenso del uso de la violencia física (“me pegan”) y de las amenazas. Estas apreciaciones se refieren a las celdas sombreadas, tomadas así del documento original.

A partir de los datos proporcionados, el documento del Defensor del Pueblo (2007:144) concluye que todos los tipos de maltrato por los que se ha indagado tienen lugar en los centros docentes de secundaria españoles, si bien cada uno de ellos con un nivel de incidencia muy distinto. Así, de acuerdo con la incidencia estimada por las alumnas y alumnos que se declaran víctimas de las diferentes modalidades de maltrato, el porcentaje más alto corresponde a los abusos por agresión verbal, cuyas diversas manifestaciones se sitúan entre el $2 \%$ y el $3 \%$, seguido de la exclusión social y de las agresiones efectuadas a través de las propiedades (en este caso, esconderlas). Con menores porcentajes de incidencia se sitúan las conductas de robo y de amenazas para intimidar, seguidas de las agresiones físicas directas (pegar) y de los destrozos de material. Un porcentaje de escolares menor al $1 \%$ alude a los chantajes (obligar a otro a hacer cosas que no desea), al acoso sexual y a las amenazas con armas. Por tanto, podría concluirse que existe una relación inversa entre la gravedad de las conductas y su nivel de incidencia, pero siempre teniendo en cuenta las precisiones que deben hacerse cuando se habla de "gravedad" en los distintos tipos de maltrato.

\subsubsection{El informe Cisneros X. Acoso y violencia escolar en España}

Aún hoy, casi una década después de su publicación, el documento Acoso y violencia escolar en España (Oñate y Piñuel, 2007), también conocido como el Informe Cisneros $X$, es un referente cuando se trata de medir el alcance del fenómeno del acoso escolar en España. El ambicioso estudio analizó una muestra de casi 25.000 alumnos de $2^{\circ}$ de Primaria hasta $1^{\circ}$ de Bachillerato de 14 comunidades autónomas². El 52 \% fueron niños y el 48 \%, niñas.

De entre los resultados del análisis cabe destacar cuestiones relativas a la incidencia del acoso escolar, a la evolución de esa incidencia según cursos así como a los comportamientos y prácticas de violencia y acoso más extendidas. Sobre la incidencia, el informe revela una tasa de acoso escolar del 24,4 \% en niños y del 21,6 \% en niñas y sitúa la tasa total en el 23,3\%. Asimismo es visible el descenso de la tasa de acoso escolar de los cursos más bajos a los más altos de tal manera que la tasa más alta se observa en $3^{\circ}$ de Primaria y la más baja en $4^{\circ}$ de ESO.

1 Quedaron fuera de la muestra la Comunitat Valenciana, la Rioja y Baleares y las ciudades autónomas Ceuta y Melilla. 
En cuanto a las prácticas, a la luz de los resultados del informe, las prácticas más habituales son las que el propio informe sitúa en la violencia verbal o psicológica: llamar por motes, no hablar, insultar o reírse de las equivocaciones, etc. Sin embargo, y según revela el propio texto, "las conductas de violencia psicológica basadas en la burla y la exclusión social son las que más incrementan el riesgo de suicidio” (Oñate y Piñuel, 2007: 28).

\subsection{Violencia en las aulas y medios de comunicación}

Observando las noticias más destacadas de los periódicos de tirada nacional y regional, Guerrero y Suárez (2012) concluyen que es poco usual leer en la prensa diferentes casos de violencia o acoso, a pesar que estos casos no solo se están dando entre adolescentes de la Educación Secundaria Obligatoria (ESO), sino que este suceso ocurre cada vez más en edades tempranas. Desde su punto de vista, para erradicar este problema es necesario "un esfuerzo para hacer llegar al ciudadano de a pie todas las noticias, por insignificante que sean, referidas a este tema con el fin de desarrollar metodologías que impliquen los valores señalados desde la Educación Primaria” (Guerrero y Suárez, 2012: 1989).

Por otra parte, Márquez y Jáuregui (2005:116) consideran que el tratamiento del tema de la violencia escolar por parte de los medios de comunicación muestra la existencia de puntos de vista divergentes sobre el hecho en función del enunciador del discurso: los políticos lo niegan, los profesores lanzan la voz de alarma y los especialistas se refieren al fenómeno como una enfermedad social. Después de analizar los diarios ABC, El País, El Diario de Sevilla, Ideal, La Vanguardia y Metro, estos autores concluyen que los representantes políticos buscan neutralizar la crítica social, especialmente en lo que se refiere a la adecuación de las medidas en política educativa. El discurso de docentes y de especialistas crea un estado de conciencia de alerta y sirve de legitimación a la idea explícitamente formulada de la necesidad de reformas. Por su parte, los especialistas apuntan hacia la necesidad de transformar el clima social que genera la violencia, concretamente el imperativo de recuperar la enseñanza en valores humanos olvidados.

El suicidio de Jokin Ceberio en septiembre de 2004 tras sufrir acoso escolar supuso, para Navarrete (2009: 336), "la aparición en la agenda de los medios de comunicación de la temática del acoso escolar”. Este autor recogió y analizó las informaciones de varios periódicos nacionales tanto inmediatamente posteriores al suicidio de Jokin como de años más tarde y considera que la prensa ha generado en este asunto una conciencia social desde la crítica y la denuncia.

Más crítico con el trabajo de los medios y de los periodistas en el tratamiento de la violencia en las aulas es el trabajo de Rodríguez referido a la difusión que hizo el periódico El Mundo del conocido como Informe Cisneros Xy la rapidez con la que se lanzaron a publicar datos ciertamente alarmantes sin preguntarse por qué otras instituciones referenciaban datos de acoso escolar más bajas. Rodríguez (2007: 28) concluía así:

La información que hemos comentado no es más que uno de tantos ejemplos diarios en que la clase periodística (o una parte de ella) realiza su trabajo de forma manifiestamente deficiente. Con excesiva asiduidad los me- 
dios se comportan como meros altavoces de informaciones a las que no se somete al imprescindible escrutinio, transfiriendo toda la responsabilidad sobre su contenido a los sujetos promotores. Especial gravedad revisten aquellos casos en que la noticia publicada se presenta bajo la forma de conocimiento científico objetivo, apuntalado por encuestas y estadísticas supuestamente incontrovertibles. La divulgación acrítica de esos mensajes contraviene la obligación ética de la prensa de contribuir a la conformación de una opinión pública informada y libre, y contribuye a instalar en la ciudadanía opiniones, actitudes y conductas fundadas en afirmaciones cuanto menos discutibles.

\section{Marco metodológico del estudio}

Partiendo de los objetivos presentados en la introducción y siguiendo los planteamientos teóricos del framingy la metodología del análisis de contenido se plantea el análisis de los textos sobre el acoso escolar publicados a lo largo del curso escolar 2014-2015 por los periódicos El Mundo y El País.

\subsection{Análisis de contenido a partir de la teoría del framing}

La técnica que se ha seguido para la realización de este estudio es el análisis de contenido a partir de la teoría del framing. Krippendorf (1990: 28) define el análisis de contenido como "una técnica e investigación destinada a formular, a partir de ciertos datos, inferencias reproductibles y válidas que puedan aplicarse a su contexto”. Con ello añade un nuevo elemento, el contexto, que sería el elemento que envuelve cualquier mensaje y sus significados. Por tanto, cualquier análisis de contenido que se lleve a cabo ha de realizarse teniendo en cuenta el contexto en el que aparecen los datos, y además basar en éste su justificación. Así, en la investigación, el hecho de situar los datos en su contexto social brinda la oportunidad de conocer su significado. Los mensajes no tienen un único significado que necesite "desplegarse". Por el contrario, existe una variedad de perspectivas para interpretar su significado, desde las palabras, asociaciones, etc. Además, Krippendorf (1990: 40-44) recoge una serie de características propias del análisis de contenido que aglutina las ideas citadas y que se resumen en las siguientes: el análisis de contenido no es una técnica intromisiva; acepta material no estructurado; 3) es sensible al contexto y por lo tanto capaz de procesar formas simbólicas; puede abordar un gran volumen de información.

Por tanto, se ha tratado de realizar una aproximación al objetivo de esta investigación, que no es otro que la búsqueda de una estructura de interpretación general de la realidad desde de la cobertura informativa del fenómeno del acoso escolar. Aquí es donde entraría en juego el concepto de encuadre o framing. Como reconocen Entman, Matthes y Pellicano (2009: 180), el análisis de encuadres en los medios de comunicación se ha convertido en una metodología viva y relevante. En esencia, el framing analiza la selección y la relevancia de ciertos aspectos de un problema mediante la exploración de imágenes, estereotipos, metáforas, actores y mensajes. Así, para Entman (1993: 52), el framing selecciona "algunos aspec- 
tos de la realidad percibida y los hace más destacados en un texto comunicativo, de tal modo que promuevan una particular definición del problema, interpretación causal, evaluación moral y/o recomendación del tratamiento". Considera que el framing es una acción estratégica, la cual genera esas cuatro consecuencias en el discurso: la definición del problema, atribución de causalidad, producción de un juicio moral y recomendación de tratamiento o solución ante el problema, en este caso, el fenómeno de la violencia entre iguales en el contexto escolar.

A pesar de que el framing es en las últimas décadas una línea teórica recurrente en la investigación en comunicación, aún se constata cierta ambigüedad teórica. En este sentido, se han realizado propuestas de delimitación o, al menos, de reorganización de cuatro décadas de estudios, como la de Vicente y López (2009:16), que se preguntan en qué estado se encuentra la investigación sobre el framing en las ciencias de la comunicación y si existen diferencias reseñables entre el contexto internacional y el español. Siguiendo sus conclusiones, en una última etapa en la que se encontraría actualmente esta perspectiva teórica se habría consolidado "una base teórica propia que comienza a ser compartida por toda la especialidad" (Íbidem: 22).

\subsection{Selección de la muestra y periodo de análisis}

Los diarios seleccionados para el estudio han sido El País y El Mundo por ser dos de las principales referencias de alcance nacional. Aunque en un caso como el que ocupa esta investigación, a priori, la línea ideológica tiene escasa influencia, se ha optado por escoger dos líneas distintas: el centro-izquierda y el centro-derecha, respectivamente, atribuidos tradicionalmente a estas dos cabeceras. La muestra para el análisis se obtuvo a través de la base de datos MyNews, que ofrece la posibilidad de disponer de la página del periódico en formato pdf, mediante la búsqueda de la referencia "acoso escolar". En total se analizaron 19 piezas publicadas en el diario El País y 31, en El Mundo.

Al tratarse de un tema de estudio vinculado al ámbito educativo se ha creído conveniente limitar el periodo de análisis a la duración del curso escolar. Sabiendo que las fechas de inicio y final del curso académico varían en cada comunidad autónoma se ha optado por establecer el análisis de los textos periodísticos publicados entre el 1 de septiembre de 2014 y el 30 de junio de 2015 para tener la posibilidad de estudiar un curso escolar completo.

\subsection{Descripción de la ficha de análisis}

Siguiendo a Igartua (2006) se ha llevado a cabo un programa de codificación que recoge una descripción de todas las variables cuantitativas y cualitativas tenidas en cuenta en la ficha de análisis según cada unidad recogida. La ficha de análisis contiene los siguientes elementos, de los cuales algunos se codifican siguiendo una lista cerrada de opciones, mientras que otros son de respuesta abierta: 
I. Medio: Se codifica el nombre del periódico en cada caso.

II. Fecha: Se incluye la fecha de la unidad de análisis que sirve tanto como para su localización como para desarrollar más adelante los gráficos de atención mediática por semanas.

III. Titular: Se copia el titular de la pieza.

IV. Sección: Aunque los medios generalistas comparten, de hecho, las mismas secciones, los nombres pueden cambiar. Así, por ejemplo, la sección con la actualidad de todo el país puede llamarse en un periódico España y en otro, Nacional; o la sección cultural denominarse Cultura o Culturas. Por este motivo, se ha optado en este caso por no ofrecer una lista cerrada de secciones y dejar la codificación libre.

V. Autoría e identificación: En primer lugar se codifica el tipo de firma, si la lleva, de la unidad de análisis. Es decir, si lleva la firma de alguien de la redacción o de alguna agencia, así como otras posibilidades. En segundo lugar se identifica al autor del texto y, en los casos que sea necesario, su cargo.

a) Redacción: tanto si se firma como Redacción o con el nombre de algún redactor.

b) Agencias: si lleva la firma de alguna agencia.

c) Colaborador: lleva la firma de un colaborador habitual (columnista).

d) Sin firma: algunas piezas no llevan firma como los breves o los sueltos de opinión o los editoriales.

e) Ciudadano: se emplea esta fórmula en las cartas al director y otras formas de colaboración ciudadana.

f) Esporádico: se codifican así las firmas de los artículos de opinión publicados, normalmente, al hilo de algún acontecimiento, suceso o temática especial. Por ejemplo, en el caso de este trabajo, un artículo de un experto en orientación o de la madre de una víctima de acoso escolar.

g) Otros: cualquier opción no contemplada anteriormente.

VI. Género: Siguiendo algunas de las muchas clasificaciones de géneros periodísticos disponibles (López, 2002: 21; López, 2007: 188; Grijelmo, 2002: 27; Parratt, 2008) se ha establecido la siguiente lista de posibilidades:

a) Análisis: piezas en las que predomina la interpretación más que la información y en las que se establecen hipótesis, causas o consecuencias sobre determinados hechos de actualidad.

b) Artículo: textos firmados por personas ajenas al medio pero que, por su reconocimiento público o su cargo, aportan su punto de vista sobre alguna cuestión de la actualidad.

c) Breve: texto de pequeña extensión, a menudo sin firma, con los elementos básicos de la noticia. Se identifican a veces porque se agrupan varios breves, por ejemplo, en una misma columna.

d) Columna: unidades con periodicidad, y a menudo ubicación, fijas en el periódico con opiniones e interpretaciones personales. 
e) Crónica: textos que relatan cronológicamente un hecho, aunque con elementos literarios o de estilo propio del que los firma.

f) Editorial: piezas en las que el periódico, como empresa o institución, pone de manifiesto su postura u opinión sobre un hecho de actualidad.

g) Entrevista: exposición de la conversación de un personaje en formato pregunta-respuesta. También se identifica como entrevista si la exposición se hace de forma narrativa.

h) Noticia: el texto informativo básico, que contiene los elementos básicos de un hecho. En el caso de la prensa escrita se caracteriza por el uso de verbos en pasado referidos, habitualmente, al día anterior.

i) Reportaje: texto informativo que incluye elementos noticiosos, declaraciones de diversas fuentes, ambiente y carácter descriptivo. Se permite en ocasiones, como la crónica, elementos literarios, por ejemplo, en la titulación.

j) Suelto: textos que, generalmente, acompañan al editorial con el mismo objetivo de plantear la opinión del medio sobre un tema, aunque de forma más breve.

k) Viñeta: ilustraciones vinculadas a la actualidad informativa con carga de interpretación u opinión.

l) Carta al director: textos que recogen la opinión de los ciudadanos sobre temas dispares.

m) Otros.

VII. Fuente y actor principal: Siguiendo en una primera lectura un análisis deductivo de las unidades recogidas se estableció una lista de actores o fuentes principales. En los casos con variedad de fuentes se identifica como principal la que aparece en mayor número. Se ha considerado la opción de equilibrio en los casos en los que no sea posible identificar alguna como principal.

a) Políticos: miembros de la administración o la clase política.

b) Profesor: clase docente.

c) Alumno: el peso del contenido del texto recae sobre el alumnado.

d) Familia: tanto de las víctimas como de los autores del acoso.

e) Expertos: fuentes que por su cargo, formación o profesión actúan como fuente especialista. Son habituales en este caso perfiles universitarios o de colectivos profesionales.

f) Víctima: las víctimas del acoso escolar son fuente o actor principal.

g) Otros: cualquier aspecto no incluido en las variables anteriores.

VIII. Encuadre: Siguiendo la propuesta de Entman (1993) ya descrita se establecen las siguientes opciones en función de la acción del texto.

a) Definición del problema: la unidad de análisis promueve una particular definición del problema. Asimismo, el texto no va más allá de describir unos hechos. 
b) Identificación de las causas: en el texto se trata de reconocer las causas o los responsables del problema.

c) Evaluación moral: las piezas promueven una evaluación moral expresa del fenómeno del acoso escolar.

d) Recomendación de tratamiento: el texto periodístico contiene propuestas de acción para el futuro o soluciones al fenómeno del acoso escolar y la violencia en las aulas.

IX. Observaciones: Se incluye aquí cualquier aspecto relevante del texto que pueda ser tenido en cuenta.

\section{Resultados del análisis}

La presentación de los resultados se hace siguiendo las variables descritas en la ficha de análisis. Se aportan, además, algunos ejemplos.

\subsection{Atención mediática}

El suicidio de Arancha en el mes de mayo de 2015 tras haber sufrido acoso escolar marca la atención mediática del asunto en los dos periódicos analizados, como es fácilmente apreciable en el gráfico 1. El diario El Mundo publica a lo largo de todo el periodo analizado 31 textos, la mayoría de los cuales corresponde a los meses de mayo (15) y junio (5) a partir de que se conoce el caso. De la misma manera sucede con el diario El País. De las 19 piezas analizadas procedentes de este periódico la mayoría corresponden a los meses de mayo (11) y junio (2). La figura descrita por las líneas del gráfico 1 muestra de manera clara el pico de atención mediática al conocerse en el mes de mayo el suicidio de la joven del instituto madrileño Ciudad de Jaén.

Es precisamente el periódico El País quien publica en primer lugar la información subrayando la condición de discapacitada de la víctima ("Una adolescente discapacitada se suicida en Madrid tras sufrir acoso escolar", El País, 23 de mayo de 2015). Al día siguiente, El Mundo publica la información citando al diario de la competencia en el texto aunque llevando al titular una declaración de la presidenta de la Asociación Contra el Acoso Escolar (ACAE), Encarna García ("Matar en la escuela sigue saliendo gratis", El Mundo, 24 de mayo de 2015). Sin embargo, este diario publica esta información, todavía, en la edición local de Madrid. La atención dura aproximadamente una semana y ambos periódicos tratan de reconstruir a partir de las declaraciones del entorno familiar y escolar la secuencia de acontecimientos que desembocan en el suicidio de Arancha ("El acosador de la niña que se suicidó": "O me das 50 euros o voy a pegarte", El País, 26 de mayo de 2015; "El infierno de Aranzazu y su acosador", El Mundo, 27 de mayo de 2015).

Por otra parte, ambas cabeceras habían publicado en septiembre de 2014 la imputación por parte de la Fiscalía de Menores del Principado de Asturias de dos adolescentes por la muerte de Carla Díaz Magnien, una niña de 14 años que se suicidó en Gijón en abril de 2013 después de meses de acoso escolar. En ambos casos la información se centraba en la re- 
acción de la madre de la víctima. ("Me decían: 'Son cosas de crías", El País, 28 de septiembre de 2014; Imputadas dos niñas por el suicidio de Carla, El Mundo, 25 de septiembre de 2014).

Tres meses después y también con la madre como protagonista se publicó la condena de las dos menores a cuatro meses de tareas socioeducativas. Entre la imputación y la condena de estas dos adolescentes destaca el amplio tratamiento que da El Mundo al caso de un niño de 15 años a quien se le reconoció por parte de la Junta de Castilla y León una minusvalía del 3\% por estrés postraumático vinculado al acoso escolar. El 4 de noviembre de 2014 el periódico El Mundo titula "Discapacidad a golpe de lapicero" un amplio reportaje de varias páginas con la historia de este caso pionero en España. En su editorial del mismo día se invitaba a reflexionar sobre el "fracaso colectivo" que supone que en el lugar donde un niño debe ser formado sufra secuelas de por vida por el maltrato sufrido.

Gráfico 1. Atención mediática

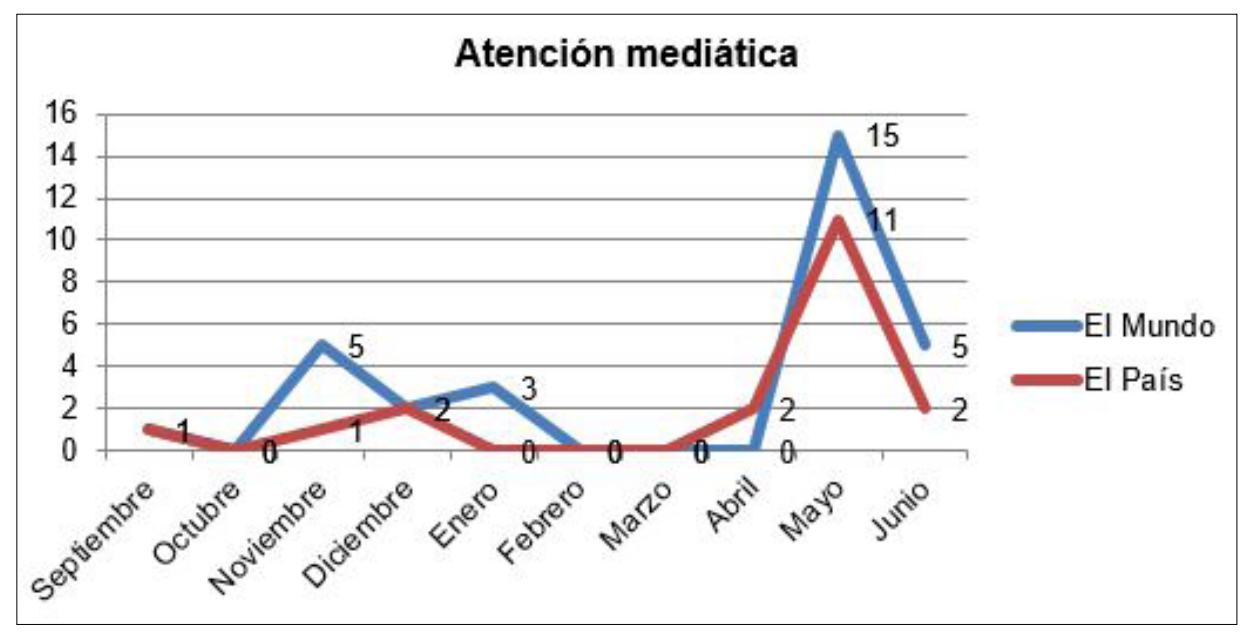

Fuente: Elaboración propia

\subsection{Sección}

Los gráficos 2 y 3 ayudan a ver cómo los textos analizados en ambos periódicos son publicados en su mayoría en las páginas de alcance nacional, aunque en diferentes secciones. Mientras que $E l$ Mundo (gráfico 2) opta preferentemente por la ubicación en la sección "Sociedad" (21), El País (gráfico 3) se decanta por las páginas de España (12). Es importante, asimismo, el número de textos que aparece en las páginas de “Opinión”. Así, en páginas de opinión El Mundo publica 7 piezas y El País, 4. 
Como se ha señalado en el apartado correspondiente a la atención mediática, destaca el hecho de que el caso de Arancha se publique en El Mundo primero en la edición regional de Madrid. Igualmente conviene señalar que esa primera vez la información se publica bajo el epígrafe de "sucesos".

Gráfico 2. Distribución por secciones en El Mundo

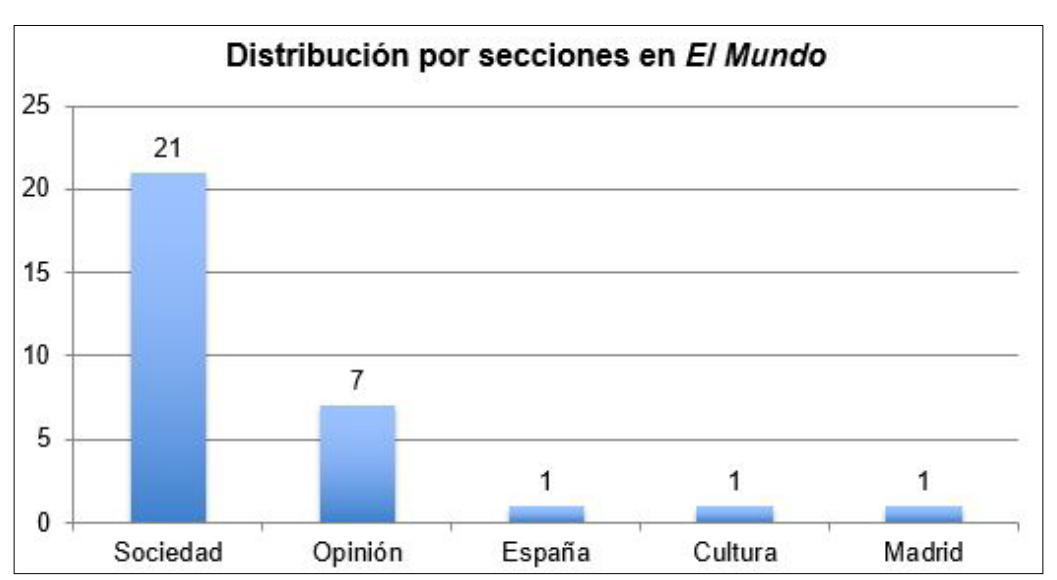

Fuente: Elaboración propia

Gráfico 3. Distribución por secciones en El País

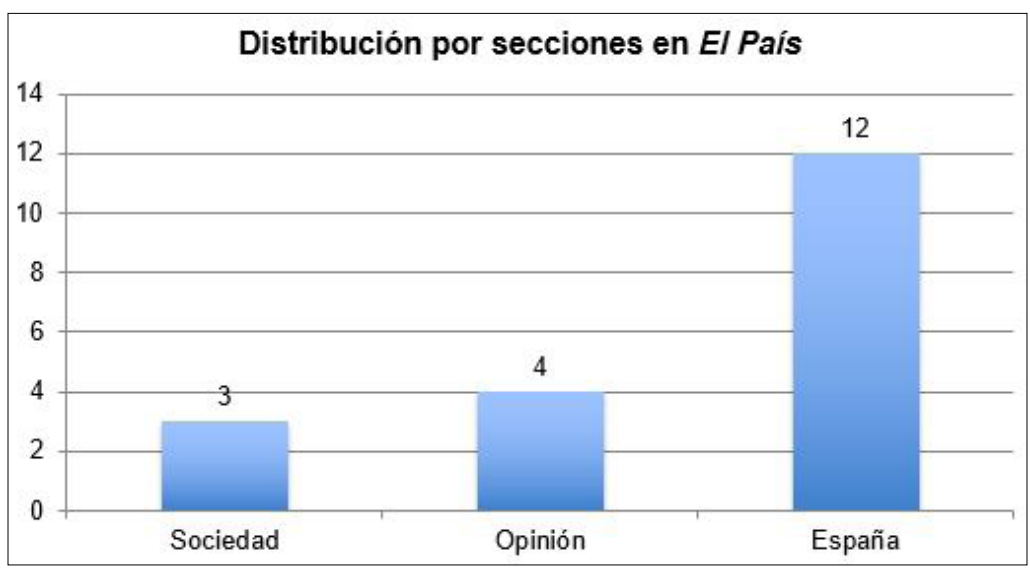

Fuente: Elaboración propia 


\subsection{Autoría de los textos}

Los textos publicados tanto por El Mundo (17) como por El País (15) aparecen en su mayoría firmados por periodistas de la redacción, como así es visible en el gráfico 4. Sin embargo, la variedad detectada en cuanto al nombre de los autores indica, en primer lugar y sobre todo al principio, que no existen periodistas especialmente asignados al asunto y, en segundo lugar, en la fase de mayor atención, el interés por proporcionar el mayor número de contenidos posible.

Por otra parte, conviene destacar la presencia de la categoría “ciudadano" tanto en El Mundo (4) como en El País (2), que se corresponden con las firmas de las cartas al director. En el diario El Mundo se publican, además, tres piezas que llevan la firma que se ha llamado "esporádico" y que son los artículos publicados expresamente relacionados con el tema por firmas ajenas a la redacción o los columnistas. También se han recogido textos sin firma (7 en El Mundo y 1 en El País) que se corresponden, algunos de ellos, a editoriales de ambos periódicos. Tanto las firmas de artículos de opinión como de editoriales se comentan en el apartado siguiente, que se refiere a los géneros periodísticos.

Gráfico 4. Autoría de los textos

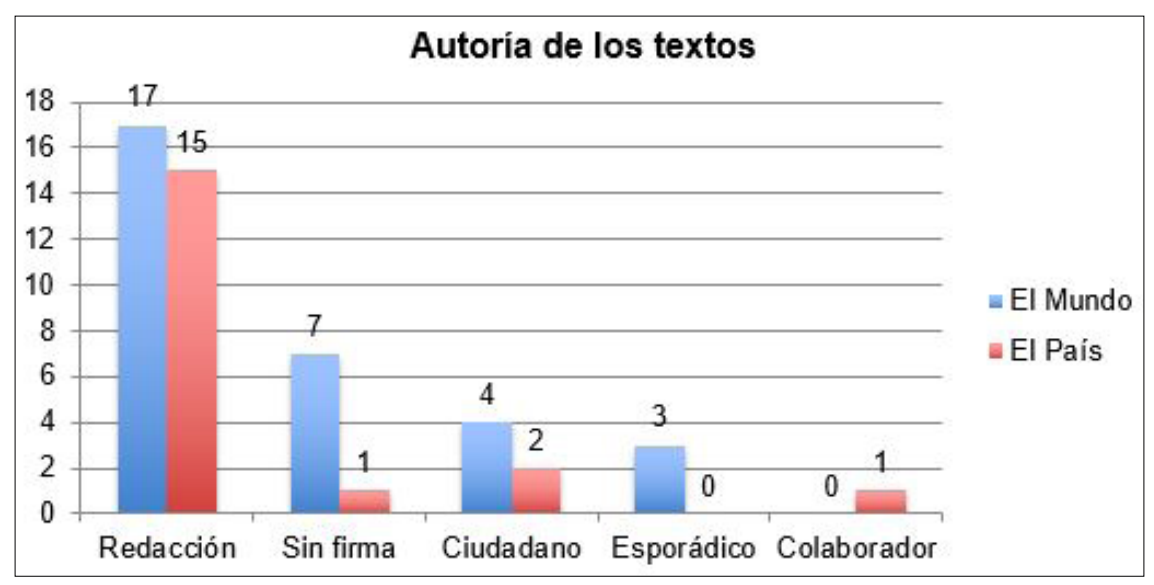

Fuente: Elaboración propia

\subsection{Géneros}

Los principales géneros considerados informativos -noticia y reportaje- son los preferidos a la hora de abordar el asunto del acoso escolar en general y, en particular, el del suicidio de la joven del instituto madrileño. No conviene olvidar que, como se ha visto en el apartado correspondiente a la atención mediática, el acoso escolar solo llega a la agenda de los medios tras la muerte de una estudiante adolescente. 
El gráfico 5 recoge esta variedad de géneros empleados por ambos periódicos. Así, el diario El País opta por la noticia para tratar la información en más de la mitad de informaciones (11) y por el reportaje en tres de las piezas analizadas. El periódico $E l M u n d o$, por su parte, da más peso a la profundización que supone el reportaje (8) una vez aportados los datos y aspectos básicos con los textos presentados como noticia (6). Se emplea el género noticia, por ejemplo, cuando se da a conocer por primera vez un hecho o se recogen novedades de un caso de mayor duración ("Wert anuncia un plan contra la violencia escolar”, El País, 22 de abril de 2015; “El expediente al director de la alumna que se suicidó indigna a los docentes”, El País, 29 de mayo de 2015; “Detenidos tres menores por acoso escolar en Madrid”, El País, 11 de junio de 2015; "Imputadas dos niñas por el suicidio de Carla”, El Mundo, 25 de septiembre de 2014; “Condenadas dos menores por acosar a Carla”, que se suicidó, El Mundo, 31 de diciembre de 2014; “La Fiscalía imputa a otro menor”, El Mundo, 28 de mayo de 2015).

Por el contrario, se opta por el género reportaje para dar mayor profundidad a un asunto en busca de causas y consecuencias y suele incluir mayor cantidad de fuentes, mayor libertad en la titulación e incluso puede contener hasta algunas dosis de creatividad en la redacción, que va más allá de la clásica pirámide invertida. Así arranca el reportaje titulado "Un polvorín en el que aprender la lección":

"Los globos aguardaban en la sala de actos junto a las orlas. En los armarios, los trajes de fiesta que tanto había costado pagar... El viernes 22 de mayo todo estaba preparado en el instituto Ciudad de Jaén para la fiesta de graduación de los alumnos de bachillerato. Tendría que haber sido un día de gloria para el barrio de Orcasur (en Usera, al sur de la capital)" (El País, 31 de mayo de 2015).

Un cierto toque literario, en este caso marcado por la primera persona, se observa igualmente en los primeros párrafos de "El infierno de Aranzazu y su acosador":

“Tenía 16 años de los difíciles, un mascarón de proa de niña introvertida, un retardo en la respuesta y una disfunción muy ligera: si no te fijabas mucho, si no te lo contaban, si no sacabas la lupa, casi ni enterabas de que la chica de $3^{\circ}$ de ESO arrastraba una pequeña discapacidad" (El Mundo, 27 de mayo de 2015).

Por otra parte, El Mundo aporta mayor variedad de géneros. Así, publica hasta cuatro artículos y tres cartas al director. Es destacable que $E l$ Mundo lleve el tema a uno de sus editoriales en tres ocasiones ("Una resolución loable que obliga a reflexionar sobre el acoso escolar", 4 de noviembre de 2014; "La lucha contra el acoso escolar, responsabilidad directa de los centros", 31 de diciembre de 2014; "El acoso escolar, una realidad oculta que se hace visible solo cuando muere un niño", 26 de mayo de 2015). Esto indica la importancia que el asunto acoso escolar adquiere para el medio en un determinado momento. En este sentido, El País lleva el tema a sus editoriales solo en una ocasión ("Afrontar el acoso escolar", 2 de junio de 2015). Como se ha apuntado, la variedad de géneros en el diario de Prisa es menor. 
Gráfico 5. Géneros empleados

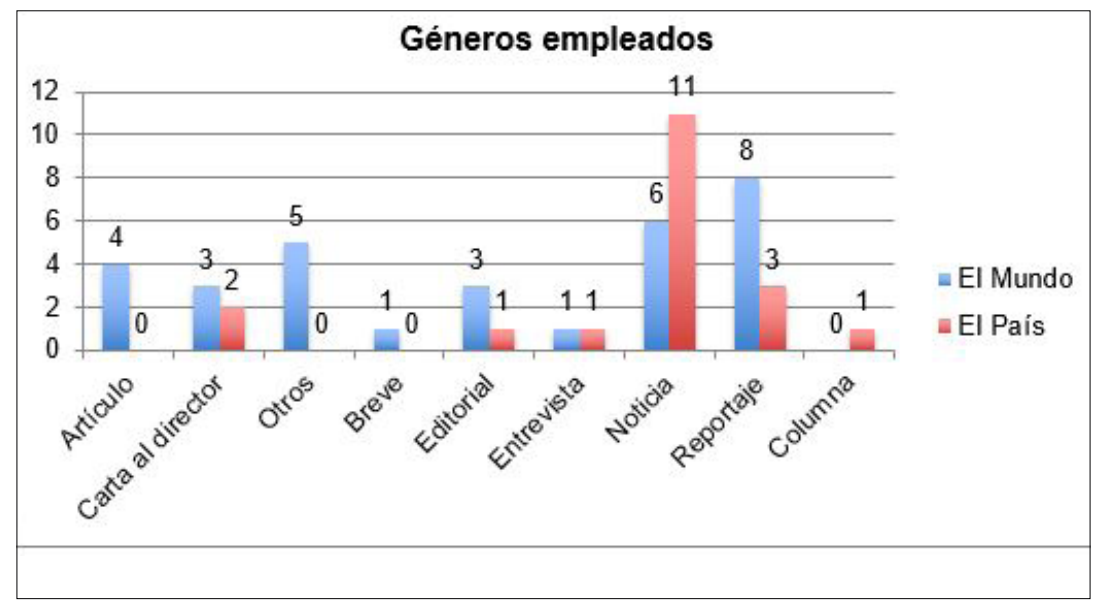

Fuente: Elaboración propia

\subsection{Fuente o actor principal}

En ocasiones no es posible o resulta difícil identificar una fuente concreta. Esto sucede especialmente en el caso de los géneros de opinión, en los que un columnista habitual o el mismo periódico a través de un editorial opina sobre el asunto, plantea un análisis o propone líneas de actuación. En estos casos se habla de actor principal puesto que si bien es cierto que no se cita ni presenta una fuente determinada sí se pone el acento, por ejemplo, en las víctimas del acoso, los alumnos, los acosadores, las administraciones o las familias.

Así, ambos periódicos acuden a variedad de fuentes y coinciden en el protagonismo de las familias -de víctimas o agresores- y los expertos (gráfico 6). En El Mundo sobresale la presencia del entorno familiar de la víctima (4) así como de expertos en la materia (5). En el diario El País, familia (5) y expertos (3) son también las principales categorías. Por el contrario, en ambas cabeceras destaca la ausencia de profesores y alumnos, testigos directos en muchos casos, de los acontecimientos.

Mediante el testimonio de los padres los diarios presentan al lector una aproximación a las últimas horas de la víctima o denuncian la inacción de los centros o las autoridades.

"Escuché un golpe en el portal. Más o menos a las 8.05 llamó una vecina: ‘ßBaja, baja!. Vi a mi hija, la mochila se había quedado en la primera planta”, cuenta Mohammed. La ambulancia se llevó a la menor” (“Denuncié el acoso, pero mi niña está muerta", El País, 28 de mayo de 2015). 
“¿Por qué no me informaron de lo que sucedía, por qué no me avisaron de lo que hacían Por qué, por qué y otra vez por qué” (“El colegio ni me avisó ni tomó medidas”, El Mundo, 2 de enero de 2015).

En este sentido, destaca la carta que Montserrat Magnien escribe al acosador de su hija en El Mundo:

"Lo primero que quiero hacer es presentarme. Soy la mamá de Carla Díaz, esa niña a la que destruyeron sus ilusiones, su futuro y lo más importante: su vida. Te conozco, matón. A mi niña la insultabais y agredíais. E incluso animabais al resto de compañeros para que se alejaran de ella. Como si fuera una persona con una enfermedad contagiosa” (“Gente como tú acabó con ella”, El Mundo, 4 de noviembre de 2014).

Así, mientras que el testimonio del entorno más directo de las víctimas ayuda mediante el relato crudo de los hechos a establecer un vínculo emocional con quien sufre el acoso, acudir a los expertos proporciona un acercamiento, aunque sea leve, a las causas de este fenómeno social así como a las propuestas para atajarlo. En este caso, se acude a asociaciones, a profesionales y a los protocolos de las comunidades autónomas en esta materia.

Un día después de conocerse el suicidio de Arancha en Madrid, la presidenta de la Confederación de Organizaciones de Psicopedagogía y Orientación de España (COPOE), Ana Cobos, se lamentaba del fracaso que supone el acoso escolar:

"En el acoso, y por eso es tan peligroso, hay uno o dos alumnos que actúan pero alrededor están los espectadores. Son los que conocen lo que ocurre y no hacen nada por cambiar la situación” "“Tenían una orientadora donde debía haber cinco", El País, 24 de mayo de 2015).

El mismo día, en las páginas de El Mundo, la presidenta de la Asociación Contra el Acoso Escolar (ACAE) proponía actuar desde la ley:

"Hay que legislar contra el acoso, para que no sea considerado una mera falta que solo se condena con trabajos sociales. Los acosadores no se reintegran en la sociedad, porque no tienen ayuda. No hay que olvidar que son personas enfermas y que además son de clase media alta, no de familias desestructuradas" "Matar en la escuela sigue saliendo gratis", El Mundo, 24 de mayo de 2015).

Ambos medios acuden en algunas ocasiones a tratar de ponerse en el lado de las víctimas. La identificación con las víctimas puede hacerse de distintas maneras, aunque este caso los dos periódicos coinciden en presentar una voz reconocida. El Mundo opta por recoger el testimonio del escritor Luis Antonio de Villena, que sufrió acoso en su infancia:

"Cuando yo le conté a mi madre las cosas que me habían ocurrido en el madrileño Colegio del Pilar entre mis 12 y 14 años -en los pasados 60- mamá se indignó y me dijo que por qué no se lo había contado entonces, porque habría tomado cartas en el asunto. Y mi madre era viva y lista. Pero yo le contesté que entonces no lo podía decir, no conseguía verbalizarlo, y que incluso, de haberlo hecho, el director -otro cura- no habría dudado en 
responder: señora, no se alarme, no ocurre nada, son chiquilladas, ellos mismos las arreglan" ("Recuerdo y teoría del acoso escolar", El Mundo, 11 de junio de 2015).

En El País, por su parte, la escritora Elvira Lindo reclama más atención a las víctimas:

"Cada cierto tiempo, el horror del acoso escolar se hace visible en la prensa porque la víctima, viéndose sin capacidad para acabar con su angustia, pone fin a su vida. Es así de crudo: sabemos de la víctima por su suicidio" (“En memoria de Carla”, El País, 4 de diciembre de 2015).

Sobresale la referencia a "otros" fuentes o actores en el periódico El Mundo (15). En ocasiones son fuente principal de la pieza textos o informes procedentes, por ejemplo de instancias judiciales ("Imputadas dos niñas por el suicidio de Carla”, El Mundo, 25 de septiembre de 2014; “La Fiscalía imputa a otra menor”, El Mundo, 28 de mayo de 2015).

Gráfico 6. Fuentes y actores

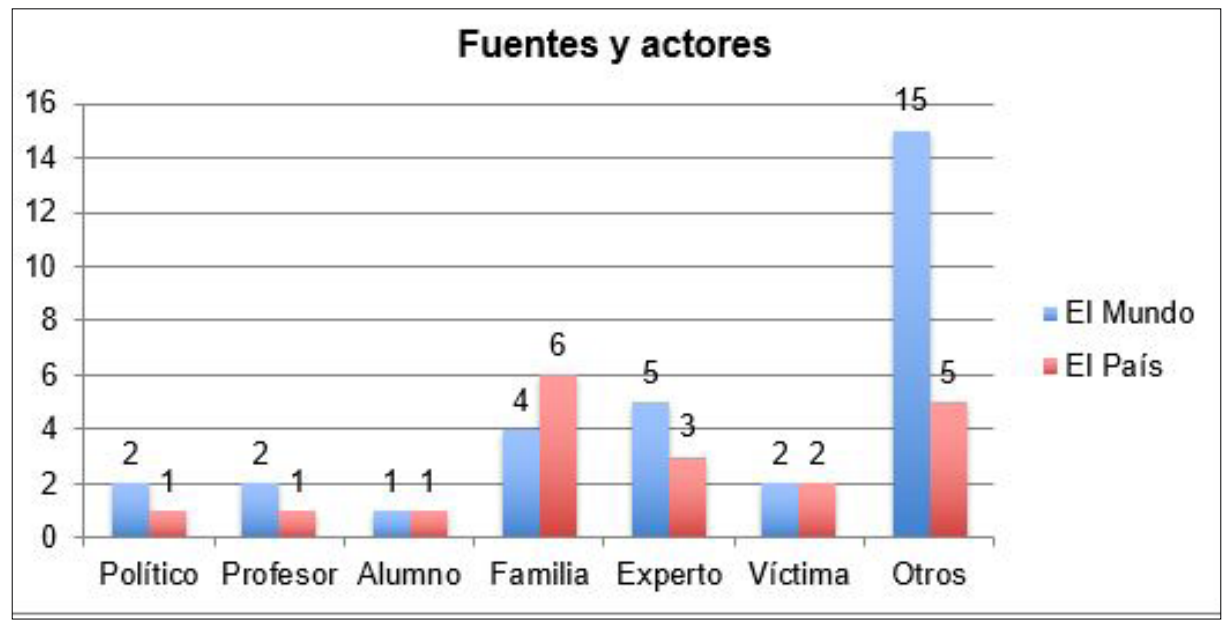

Fuente: Elaboración propia

\subsection{Encuadres}

Buena parte de los textos periodísticos analizados centran su contenido, por un lado, en la definición y descripción de los acontecimientos y, por otro lado, en las propuestas o recomendaciones de tratamiento (gráfico 7). Así, en el diario $E l$ Mundo se han identificado 13 piezas en el primer caso y 11 en el segundo. En El País, 10 y 7, que se corresponden con la práctica totalidad, puesto que solo dos se han identificado como textos que tratan de ahondar en las causas del fenóme- 
no. El Mundo, por su parte, presenta hasta en cuatro ocasiones relatos que intentan bucear en el porqué de la cuestión. Correspondientes a este diario se han señalado tres textos que contienen lo que se ha denominado una evaluación moral, principalmente, por el lenguaje empleado, muchas veces de condena.

Como se ha señalado, la mayoría de los textos no van más allá de presentar un relato más o menos fiel de la realidad. Como mucho, se llega a presentar el cómo se ha llegado al suicidio de una menor como suceso concreto pero sin llegar a entrar de lleno en las causas o razones que llevan a un adolescente a ejercer de manera continuada violencia física o verbal sobre un compañero.

"Estoy cansada de vivir", escribió la menor en un mensaje de teléfono a sus amigas antes de arrojarse al hueco de las escaleras en la vivienda familiar que compartía con su hermano menor y sus padres en el barrio de Usera, al sur de Madrid capital. Ocurrió poco antes de las nueve de la mañana de ayer, cuando en el instituto al que asistía estaban empezando las clases un día más" ("Una adolescente discapacitada se suicida en Madrid tras sufrir acoso escolar", El País, 23 de mayo de 2015).

El encuadre que se ha denominado recomendación de tratamiento es más propio de los días posteriores al estallido del caso, cuando la vorágine de las primeras horas y de querer conocer y proporcionar detalles da paso a las voces expertas que proponen en qué línea trabajar.

"Frenarlo desde sus primeros gestos, que el acoso no coja cuerpo" es la exigencia fundamental que, ayer, manifestó la Asociación Madrileña contra el Acoso Escolar (AMACAE). "Demandamos que a partir del curso que viene se cree un protocolo de prevención donde se evalúe el acoso escolar y la violencia en las aulas con una política de tolerancia cero" (“Contra 'la ley del silencio', El Mundo, 4 de junio de 2015).

En menor medida, y a pesar de tratarse de un tema que genera fuerte interés social, se han detectado encuadres denominados de evaluación moral, al menos expresada de manera explícita. En el texto citado anteriormente de Luis Antonio de Villena ("Recuerdo y teoría del acoso escolar", El Mundo, 11 de junio de 2015), el escritor juzgaba como "injusto y soez que se vayan de rositas los energúmenos que acosan".

En el caso concreto estudiado, si bien sí se han hallado los relatos de cómo los casos de Arancha o Carla llevaron a que se quitaran la vida -se relatan los insultos, las vejaciones, sus comportamientos- en menor medida se detecta mayor profundidad y examen acerca de las razones o causas que desembocan o provocan la práctica del acoso. De nuevo Encarna García, de la Asociación Contra el Acoso Escolar (ACAE) propone su diagnóstico:

"No hay vigilancia ninguna, lo que ayuda al acoso, a los insultos, a las agresiones y al aislamiento social que tanto mata. Porque una gran parte de los suicidios por acoso escolar los causa el aislamiento social, por estar 
solos/as en esa selva en la que se convierten los colegios para muchos niños" ("La conjura de los necios", $E l$ Mundo, 26 de mayo de 2015).

Gráfico 7. Encuadres noticiosos

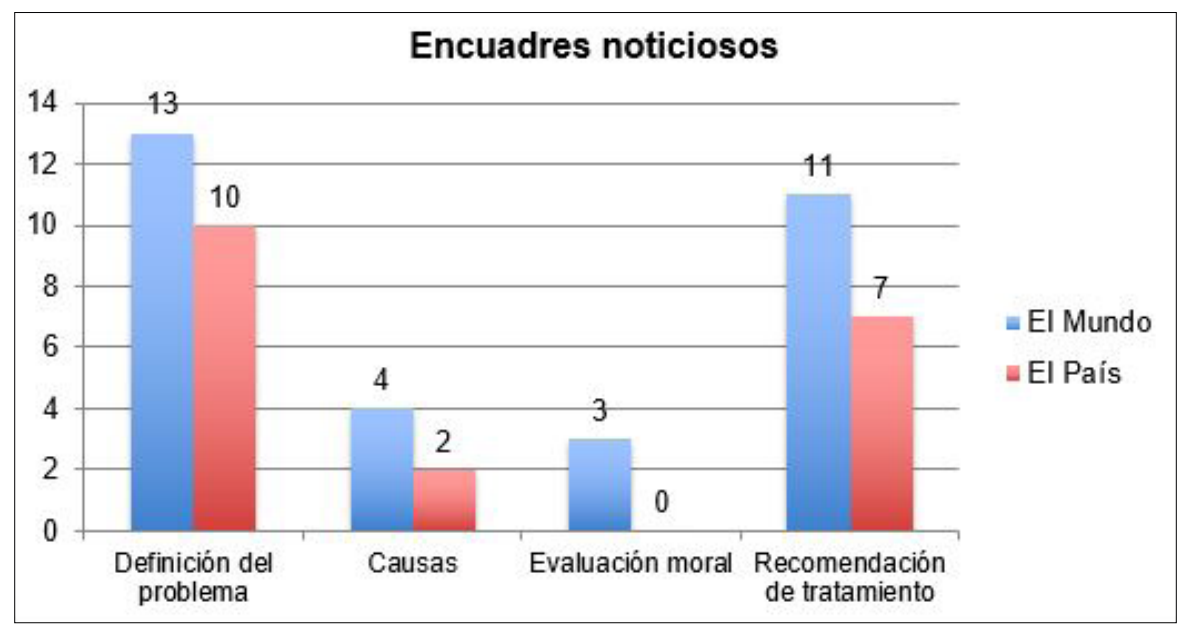

Fuente: Elaboración propia

\section{Conclusiones}

El acoso escolar es permanente, actual y está cada vez más presente en los medios de comunicación. El análisis de los mensajes periodísticos a partir de la teoría del framing ha servido y sirve para analizar la representación mediática de multitud de asuntos aunque el acoso escolar solo ha sido abordado de este modo en unas pocas ocasiones, de ahí la justificación de esta propuesta de estudio.

Coincidiendo con los planteamientos teóricos presentados en el papel de los medios de comunicación como creadores de representaciones de fenómenos y realidades es pertinente preguntarse cuál es el dibujo que se está haciendo en los medios del fenómeno del acoso escolar, a qué fuentes se recurre para examinarlo y, en definitiva, cuál es la imagen de esta realidad que llega a la sociedad. Todo esto, siendo conscientes de algunas limitaciones de esta propuesta de estudio como la limitación temporal o la profundidad del análisis. Será interesante ampliar el periodo de estudio así como analizar la relación directa entre las fuentes del discurso y los encuadres detectados. 
Por esta razón, el análisis del discurso periodístico del acoso escolar propuesto en esta investigación abre la puerta a otras líneas de estudio. Sería interesante, en primer lugar, confirmar la hipótesis de la atención mediática condicionada por un suceso trágico planteando un estudio similar que no se limite a un curso escolar. Igualmente, sería deseable aportar la perspectiva penal tras detectarse que en algunos casos se imputa directamente a los sujetos la condición de acosador o maltratador.

El análisis de contenido a partir de los planteamientos teóricos del framing se ha mostrado adecuado y pertinente para el estudio del tratamiento del acoso escolar en los diarios El Mundo y El País a lo largo del curso escolar 2014 - 2015. En concreto y siguiendo los objetivos y las preguntas de investigación planteadas al comienzo se presentan las siguientes conclusiones:

1. El suicidio de Arancha, estudiante en el instituto Ciudad de Jaén de Madrid, en mayo de 2015 condiciona la atención prestada por los diarios El Mundo y El País al fenómeno del acoso escolar durante el periodo analizado. A pesar de que en sus editoriales las propias cabeceras califican esta realidad como un fracaso social, la permanencia y la continuidad de este problema en el contexto educativo no tiene respuesta en forma de tratamiento mediático continuado.

En contraste, la reflexión del asunto en los editoriales de los periódicos analizados confirma el hecho de que se está ante un fenómeno social que preocupa. Este trabajo sigue así la línea de otros estudios (Guerrero y Suárez, 2012) en la constatación de la poca presencia en los medios de comunicación de un fenómeno como el del acoso escolar definido por expertos y administraciones como continuado y permanente.

2. La importante presencia de fuentes que se corresponden con el entorno familiar de las víctimas del acoso genera, por su parte, un discurso basado en la declaración más sentimental que racional. Es cierto, por otra parte, que el discurso del entorno de la víctima favorece la identificación del lector pero es igualmente fácil que el discurso caiga así en un dramatismo más interesado en la consecución de audiencias y difusión que en la aportación de las claves informativas. Las voces expertas, que desde su conocimiento y experiencia, aportan serenidad y rigor a un debate necesario acerca de las causas profundas que desembocan en el acoso escolar no en un desgraciado caso concreto- quedan relegadas a un segundo plano en favor del discurso del drama.

Estas apreciaciones constatan las posturas de otros planteamientos sobre las fuentes y el tipo de mensaje que transmiten (Márquez y Jáuregui, 2005). De nuevo, los docentes dan la voz de alarma y los especialistas aportan las claves. Este trabajo añade a esta consideración el papel del entorno de las víctimas de acoso, que promueve la identificación y la empatía en el camino para lograr mayor respuesta y consideración social.

3. Relacionado con esto, es destacable la ausencia de un discurso sobre el acoso escolar que profundice en las causas del fenómeno. Si bien las informaciones sobre el acoso escolar no pueden dejar de aportar las claves informativas del hecho, 
este fenómeno social requeriría de una cobertura periodística más profunda, no condicionada por los acontecimientos más desgraciados que favorecen que el discurso se quede en lo más propio de una sección de sucesos. Por el contrario, sí cabe reconocer la presencia de un discurso de lo que se ha llamado la recomendación del tratamiento, esto es, informaciones y opiniones que aportan o tratan de aportar algunas claves sobre cómo afrontar desde todos los contextos posibles esta problemática.

\section{Referencias bibliográficas}

Armero, P., Bernardino, B., y Bonet de Luna, C. (2011): “Acoso escolar.” Pediatría de Atención Primaria, vol. 13, n. 52, pp. 661-670. Recuperado de: http://doi.org/10.4321/S1139-76322011000600016.

Cepeda, E., y Caicedo, G. (2013): “Acoso escolar: caracterización, consecuencias y prevención.” Revista Iberoamericana de Educación, vol. 61, nº 3 .

Comunidad de Madrid. (2012): "Orientaciones para la prevención, detección y corrección de las situaciones de acoso escolar en los centros docentes no universitarios de la Comunidad de Madrid.” Recuperado de: http:/ /www.madrid.org/ dat_sur/site/convivencia/acoso_escolar/ACOSO_ESCOLAR_Orientaciones_generales.pdf

Defensor del Pueblo. (2007): "Violencia Escolar: El Maltrato entre Iguales en la Educación Secundaria Obligatoria 1999-2006".

Entman, R. M. (1993): "Framing: Towards Clarification of a Fractured Paradigm Framing: Toward Clarification of a Fractured Paradigm." Journal of Communication Copyright Journal of Communication, vol, 43, nº 434, pp. 51-58.

Entman, R., Matthes, J. y Pellicano, L. (2009): “Nature, sources and effects of news framing”, en K. Wahl-Jorgensen \& T. Hanitzsch (eds.), The handbook of journalism studies. Nueva York: Routledge.

Fernández, M., y Suárez, M. (2012): "El escaso reflejo que todavía tiene en los medios de comunicación la imagen corporal de las chicas y el acoso escolar. Cuando se trata de algo más que causa y efecto", en I Congreso Internacional de Comunicación y Género. Sevilla. Recuperado de: http://doi.org/10.1017/CBO9781107415324.004.

Fiscalía General del Estado. (2005): “Instrucción 10/2005 sobre el tratamiento del acoso escolar desde el sistema de justicia juvenil”. Recuperado de: https://www.fiscal.es/fiscal/PA_WebApp_SGNTJ_NFIS/descarga/MN_Instruccion10_2005. pdf?idFile=934611d6-6f25-49de-8d2a-916cad86b3ee

Grijelmo, Á. (2002): El estilo del periodista. Madrid: Taurus.

Krippendorff, K. (1990): Metodología del análisis de contenido. Teoría y práctica. Barcelona: Paidós Comunicación. 
López, A. (2002): Géneros periodísticos complementarios. Sevilla: Comunicación Social.

López, M. (2007): Cómo se fabrican las noticias. Barcelona: Paidós Comunicación.

Márquez, M., y Jáuregui, I. (2005): “La violencia escolar en los textos periodísticos”, Revista Iberoamericana de Educación, vol. 38, pp. 105-119.

Mora-Merchán, J. A., Ortega, R., Calmaestra, J., y Smith, P. K. (2010): “El uso violento de la tecnología: el cyberbullying”, en R. Ortega (Ed.), Agresividad injustificada, bullying y violencia escolar, (pp. 189-210). Madrid: Alianza Editorial.

Morduchowicz, R. (2003): "El sentido de una educación en medios". Revista Iberoamericana de Educación, vol. 32, pp. $35-47$.

Navarrete-Galiano, R. (2009): "Los medios de comunicación y la concienciación social en España frente al acoso escolar." Estudios Sobre El Mensaje Periodístico, vol. 15, pp- 335-345.

Oñate, A., y Piñuel, I. (2007): “Informe Cisneros X. Acoso y violencia escolar en España”, Instituto de Innovación Educativa y Desarrollo Directivo.

Parratt, S. F. (2008): Géneros periodísticos en prensa. Quito: Ediciones Ciespal.

Rodríguez, R. (2007): La propagación mediática del miedo. El caso del macroinforme sobre el acoso escolar. Espéculo. Revista de Estudios Literarios, $\mathrm{n}^{\circ} 37$.

Rubio, M. (2013): "Estudio sobre la percepción del profesorado en Educación Secundaria Obligatoria del acoso escolar". Revista de Educación Social, no 16.

Vicente, M., y López, P. (2009): “Resultados actuales de la investigación sobre framing. sólido avance internacional y arranque de la especialidad en España”, Zer: Revista de Estudios de Comunicación, vol, 14, nº 26, pp. 1137-1102. 